\title{
CALIBRAÇÃO GEOMÉTRICA EM ÓRBITA DO SENSOR HRC-CBERS
}

\section{B}

\section{On orbit geometric calibration of HRC-CBERS $2 B$ sensor}

\author{
Tiago Lima Rodrigues ${ }^{1,2}$ \\ Marcela do Valle Machado 1 \\ Paula Debiasi ${ }^{1}$ \\ Edson Aparecido Mitishita ${ }^{2}$ \\ Luiz Danilo Damasceno Ferreira ${ }^{2}$ \\ 1 Universidade Federal Rural do Rio de Janeiro - UFRRJ.Instituto de Tecnologia, Departamento de \\ Engenharia. BR 465, km 7, 23890-000, Seropédica, RJ, Brasil \\ 2 Universidade Federal do Paraná - UFPR.Curso de Pós-Graduação em Ciências Geodésicas.Caixa \\ Postal 515 - 12201-970 - Curitiba - PR, Brasil
}

\section{Resumo:}

A extração de informações geoespaciais de uma imagem orbital requer a orientação interior e exterior da mesma. Os parâmetros de orientação exterior (POE) podem ser obtidos de forma direta, utilizando sensores GNSS/INS e estelares, ou indireta, utilizando feições de controle no espaço objeto. Os parâmetros de orientação interior (POI) são inicialmente obtidos por calibração em laboratório antes do lançamento do satélite, e podem também ser estimados periodicamente pela calibração geométrica em órbita. $\mathrm{O}$ objetivo deste trabalho foi realizar a calibração geométrica em órbita do sensor HRC-CBERS 2B. Para tanto, foram consideradas as correções dos efeitos sistemáticos causados pela geometria do plano focal do sensor e pelo sistema de lentes do mesmo. Uma densa quantidade de pontos de controle e verificação foi utilizada, sendo que a análise da exatidão planimétrica nos pontos de verificação foi aplicada para avaliar a calibração. Quatro experimentos foram realizados analisando três diferentes conjuntos de POI. Os resultados dos experimentos de calibração foram muito próximos, não apresentando diferenças significativas. Uma maior exatidão foi obtida quando se empregou o grupo de POI modelando a translação, rotação, fator de escala e flexão das matrizes de CCD (Charge-Coupled Device), juntamente com a distorção radial simétrica do sistema de lentes.

Palavras-chave: modelo UCL, câmara HRC, calibração geométrica em órbita.

\begin{abstract}
:
The geospatial information extraction from orbital images requires the internal and external orientation. The external orientation parameters (EOPs) can be obtained directly from GNSS/INS and star sensors, or indirectly by control features in the object space. The interior orientation parameters (IOPs) are first determined from laboratory calibration process prior the satellite launch, and can be estimated by the periodically on-orbit geometric calibration. This paper aimed to perform the on-orbit geometric calibration of the HRC-CBERS 2B sensor. The corrections of systematic effects caused by the focal plane of the sensor and by lens system were
\end{abstract}


considered in the calibration process. A dense number of ground control and check points are used, and the accuracy analysis of the planimetric checkpoints was then applied to evaluate the calibration. Four experiments are performed analyzing three different groups of IOPs. The results obtained from calibration experiments were similar and did not shown significant differences. A slightly highest accuracy was obtained when the group of IOPs was used considering the translation, rotation, scale factor and bending of the CCD chips, with symmetric radial distortion of the lens system.

Keywords: UCL model, HRC camera, on orbit geometric calibration.

\section{Introdução}

As imagens obtidas por plataformas orbitais são amplamente utilizadas como fonte de aquisição de dados geoespaciais. Entretanto, para a extração das informações, é necessário que as mesmas sejam orientadas com relação a um Sistema de Referencia do espaço objeto. Os modelos de orientação são denominados de empíricos quando não são conhecidas as informações referentes aos parâmetros de aquisição do sistema, sendo que os parâmetros utilizados para relacionar o espaço imagem com o objeto não possuem significado físico (Toutin, 2004). No método denominado de rigoroso (Toutin, 2004), esta orientação é realizada com o conhecimento dos parâmetros de orientação exterior (POEs) e dos parâmetros de orientação interior (POIs), sendo integrados nas equações de colinearidade, o que viabiliza o relacionamento do ponto imagem com o ponto objeto no instante que a imagem foi obtida.

A estimativa dos POEs pode se dar de forma direta a partir das observações de rastreio de órbita e/ou de medidas com receptor (es) GNSS (Global Navigation Satellite System) embarcado (s) no próprio satélite, para a determinação da posição; e sensores INS/estelares para a determinação dos ângulos de atitude da plataforma. Caso não haja a disponibilidade dos dados de tais equipamentos, os POEs podem ainda ser estimados de forma indireta a partir de feições de controle no espaço objeto (pontos, retas e polígonos) associadas a um modelo funcional específico. Por outro lado, os POIs são estimados a partir de processos de calibração em laboratório, previamente ao lançamento do satélite, e podem também ser atualizados periodicamente após o satélite estar em órbita.

Uma vez que os POIs possibilitam a reconstrução da geometria do feixe de raio no instante da formação da imagem, as particularidades ou condições físicas da geometria interna do sensor devem ser consideradas para a obtenção de resultados acurados. Tal procedimento deve ocorrer principalmente em aplicações de georeferenciamento direto, onde os POEs são fixados no ajustamento, impedindo que as imprecisões de alguns POIs sejam compensadas pelos POEs calculados (Fototriangulação convencional).

Uma importante característica na geometria do plano focal é que na maioria dos sensores embarcados nas plataformas orbitais há um arranjo de constituição em linha CCD com múltiplas matrizes, como por exemplo IRS-1C, IKONOS, Quickbird, SPOT 5, PRISM-ALOS e HRC-CBERS 2B. Jacobsen (2005) cita que o uso de uma combinação de linhas CCD mais curtas ao invés de uma linha CCD longa ocorre devido a certas características requeridas, porém o autor não apresenta tais características. Baltsavias et al. (2006) afirma que a montagem de um arranjo de três matrizes de CCD ao invés de uma única para o sensor IKONOS pôde estar relacionada com a disponibilidade de componentes para a montagem da matriz única no momento da produção do sensor; ou por razões de velocidade de produção, custo e confiabilidade do produto. $\mathrm{O}$ autor ainda ressalta que o motivo da concepção do sensor utilizando três matrizes de CCD não é amplamente conhecida. Ainda de acordo com Jacobsen (2005), as matrizes de CCD devem ser exatamente alinhadas ou no mínimo paralelas, sendo as posições conhecidas no plano focal. Este último é necessário, pois em geral o Ponto 
Principal (PP) também não é coincidente com o centro geométrico do arranjo de matrizes (Kocaman, 2007).

Por motivos de imprecisão de fabricação, pequenos deslocamentos bidirecionais e rotações entre as matrizes de CCD no plano focal acabam ocorrendo (Poli, 2005). Além disso, os tamanhos das matrizes podem ser ligeiramente diferentes, e o afastamento da linearidade das mesmas pode também ocorrer. Neste caso, antes do lançamento do satélite é realizada a calibração geométrica para a estimativa dos valores. Segundo Baltsavias et al. (2006) e ainda Jacobsen (2005), dois problemas que contribuem para a instabilidade dos valores calibrados em laboratório, são as acelerações e mudanças ambientais drásticas impostas durante o lançamento do satélite, e a influência termal do sol quando o satélite encontra-se em órbita. Consequentemente, em alguns casos uma calibração geométrica em órbita é requerida de tempos em tempos para a estimativa de parâmetros mais significativos com a realidade física no instante da formação da imagem.

Particularmente no caso do sensor HRC do satélite CBERS 2B, o plano focal é constituído de três matrizes de CCD, sendo as duas matrizes laterais alinhadas entre si e deslocadas da matriz central em 26 mm na direção da trajetória (Epiphanio, 2009; Marcato Junior et al., 2011). Uma sobreposição de 8,5 pixels entre as imagens adquiridas por cada matriz é garantida para que haja a formação de uma imagem única que é posteriormente disponibilizada para os usuários. Desta forma, para a orientação destas imagens, todos os parâmetros discutidos anteriormente devem ser considerados para aumentar a exatidão dos resultados obtidos. Experimentos considerando alguns dos parâmetros citados podem ser vistos em Yue et al. (2009) e Zhang e Zheng (2012).

Neste contexto, o objetivo principal deste trabalho foi realizar a calibração geométrica em órbita do sensor HRC. Primeiramente foram utilizados todos os POIs considerados em Zhang e Zheng (2012), e posteriormente foram incluídas as distorções radiais simétricas do sistema de lentes, os fatores de escala e os afastamentos de linearidade das matrizes de CCD (flexões). Para a calibração geométrica foi empregado o modelo rigoroso de colinearidade, utilizando pontos como controle, juntamente com o modelo de plataforma UCL (Michalis, 2005). Uma densa distribuição de pontos de controle e de verificação sobre a imagem foi utilizada para a realização de quatro experimentos, considerando o uso de três diferentes grupos de POIs.

\section{Sistemas de referência no espaço imagem}

O primeiro sistema relacionado às imagens é o denominado Sistema de Referência de Imagem (SRI). Este é associado à matriz bidimensional dos pixels da imagem em um sistema de coordenadas coluna (C) e linha (L). A origem do sistema encontra-se ou no centro do primeiro pixel da imagem, ou no canto superior esquerdo deste, sendo a segunda a situação mais comum. Conforme ilustrado na Figura 1 (a), a direção do eixo L é coincidente com a direção das colunas da imagem, enquanto que a direção do eixo $\mathrm{C}$ coincide com a direção das linhas.

Outro sistema utilizado é o Sistema de Referência da Matriz de CCD (SRM), sendo bidimensional e com origem no centro geométrico da matriz de CCD. O eixo $\mathrm{x}_{\mathrm{s}}$ é coincidente com a direção perpendicular à linha da imagem (eixo L) e a direção do eixo ys é paralela a esta (Figura 1 (b)). 


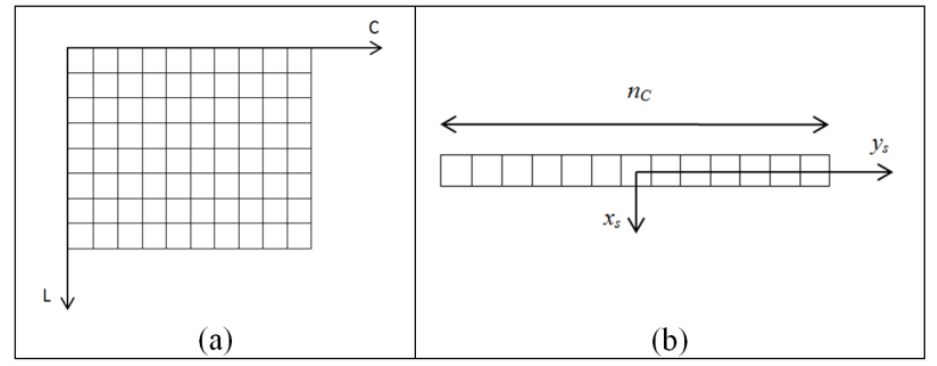

Figura 1: (a) Sistema de Referência de Imagem (SRI) (b) Sistema de Referência da Matriz de CCD (SRM).

As Equações 1 e 2 permitem a transformação do SRI para o SRM, no caso de ser um sensor de varredura linear com arranjo de constituição em linha, assumindo a situação ideal onde não existam distorções provenientes do sistema de lentes e das imperfeições das matrizes de CCD no plano focal.

$$
\begin{gathered}
x_{S}=P S \cdot L-\operatorname{int}(L) \cdot P S+0,5 \cdot P S=[L-\operatorname{int}(L)+0,5] \cdot P S, \\
y_{S}=P S \cdot C-\frac{\left(n_{C}-1\right)}{2} \cdot P S=\left(C-\frac{\left(n_{C}-1\right)}{2}\right) \cdot P S,
\end{gathered}
$$

onde $x_{s}$ e $y_{s}$ são as coordenadas do SRM, $P S$ é o tamanho do pixel na matriz de CCD em mm (considerando um pixel quadrado) e $n_{C}$ o número de colunas da imagem referente à matriz de CCD em questão.

Dispondo-se das medidas no SRM, utiliza-se posteriormente o Sistema de Referência da Câmera (SRC) para efetuar a orientação das imagens. Este é um sistema de coordenadas tridimensional, com as direções do eixo $x_{c}$ e $y_{c}$ paralelas às direções dos eixos $x_{s}$ e $y_{s}$ do SRM, respectivamente. A direção do eixo $z_{c}$ é definida de forma a se ter um sistema dextrógiro (Poli, 2005). A origem deste sistema é localizada no Centro Perspectivo (CP) do sensor que é equivalente ao ponto nodal posterior da objetiva da mesma. A projeção do CP no plano focal é denominada de PP. A Figura 2 ilustra o SRC e o SRM para um plano focal com 3 matrizes de CCD alocadas.

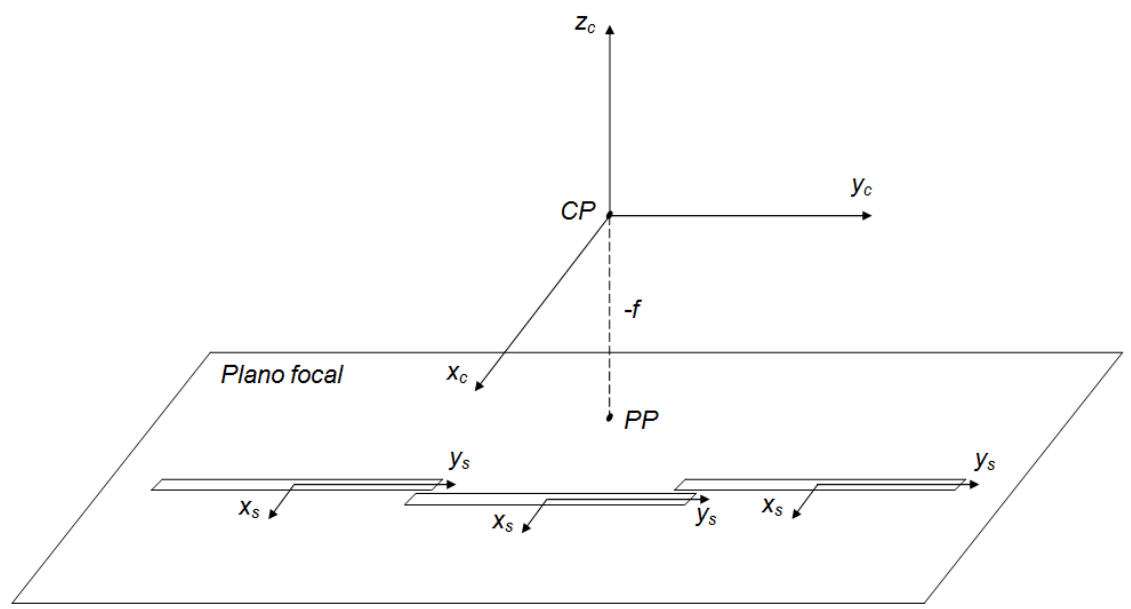

Figura 2: Sistema de Referência da Câmera e da matriz de CCD.

Considerando um caso ideal de uma matriz de CCD isenta de distorções, a transformação entre o SRM e o SRC é apresentada na Equação 3. Uma vez que em geral o PP não coincide com a posição central da (s) matriz (es) de CCD (Figura 2), uma translação bidimensional é necessária para compatibilizar a origem dos dois sistemas. 


$$
\left[\begin{array}{l}
x_{c} \\
y_{c} \\
z_{c}
\end{array}\right]=\left[\begin{array}{c}
x_{s} \\
y_{s} \\
-f
\end{array}\right]+\left[\begin{array}{c}
d x \\
d y \\
0
\end{array}\right],
$$

onde $x_{c}$ e $y_{c}$ são as coordenadas do SRC e, $d_{x}$ e $d_{y}$ são as translações do centro de uma dada matriz de CCD para o PP no plano focal.

\section{O satélite CBERS e o sensor HRC}

O programa de satélites Sino Brasileiro de Recursos Terrestres, denominado CBERS (ChinaBrazil Earth Resoucers Sattelite), foi desenvolvido em uma parceria do governo brasileiro com o chinês. Dos quatro satélites até então lançados, três (CBERS 1, 2 e 2B) obtiveram êxito em suas missões. A política de disponibilização gratuita das imagens da série CBERS no site do Instituto Nacional de Pesquisas Espaciais (INPE) disseminou o uso de dados de Sensoriamento Remoto no Brasil. Com o lançamento do CBERS 2B, satélite equipado com uma câmara de alta resolução espacial - HRC (com Ground Sample Distance - GSD de 2,5 m), viabilizou-se a utilização dessas imagens em aplicações de engenharia com necessidades de maiores resoluções espaciais.

Apesar desse satélite ter sido desativado em 2010, verifica-se ser ainda importante o desenvolvimento de metodologias para a correção geométrica destas imagens de alta resolução espacial, uma vez que muitos usuários ainda utilizam-se destas para a produção de dados geoespaciais. Além disso, existe a expectativa futura do emprego de imagens do satélite CBERS 4, que foi equipado com câmara de alta resolução - GSD de $5 \mathrm{~m}$ na banda pancromática (Inpe, 2014). Na Tabela 1 são apresentadas as principais características do sensor HRC do CBERS $2 B$.

Tabela 1: Características do sensor HRC-CBERS 2B.

\begin{tabular}{cc}
\hline Distância focal & $3398 \mathrm{~mm}$ \\
\hline Tamanho do detector & $0,010 \mathrm{~mm}$ \\
\hline Banda espectrais & $0,5-0,8 \mu \mathrm{m}$ - pancromática \\
\hline Campo de Visada & $2,1^{\circ}$ \\
\hline Resolução Espacial & $2,5 \mathrm{~m}$ \\
\hline Largura da faixa imageada & $27 \mathrm{~km}$ (nadir) \\
\hline Resolução Temporal & 130 dias \\
\hline Quantização & 8 bits \\
\hline
\end{tabular}

Fonte: Inpe (2014) e Marcato Júnior e Tommaselli (2013).

O plano focal do sensor HRC foi composto por três matrizes de CCD, cada uma contendo 4096 elementos, imageando $9 \mathrm{~km}$ na superfície terrestre, conforme representação gráfica mostrada na Figura 3. Segundo Epiphanio (2009), as duas matrizes laterais (1 e 3) eram nominalmente alinhadas entre si. Já a matriz central (2) era deslocada das demais em $26 \mathrm{~mm}$ no sentido de deslocamento do satélite na órbita (eixo $x_{c}$ do SRC). As imagens adquiridas por cada matriz separadamente são alinhadas e juntadas posteriormente para formarem uma imagem final com 12246 pixels. Para tanto, uma zona de sobreposição de 8,5 pixels em cada junção de matriz foi projetada. Além disso, um movimento lateral do satélite tinha que ser conjugado ao movimento 
longitudinal descendente do satélite de modo a compensar a rotação da Terra durante o imageamento e para que as três matrizes pudessem ser justapostas durante a construção da imagem final (Epiphanio, 2009). Este movimento é denominado de movimento caranguejo (crab movement).

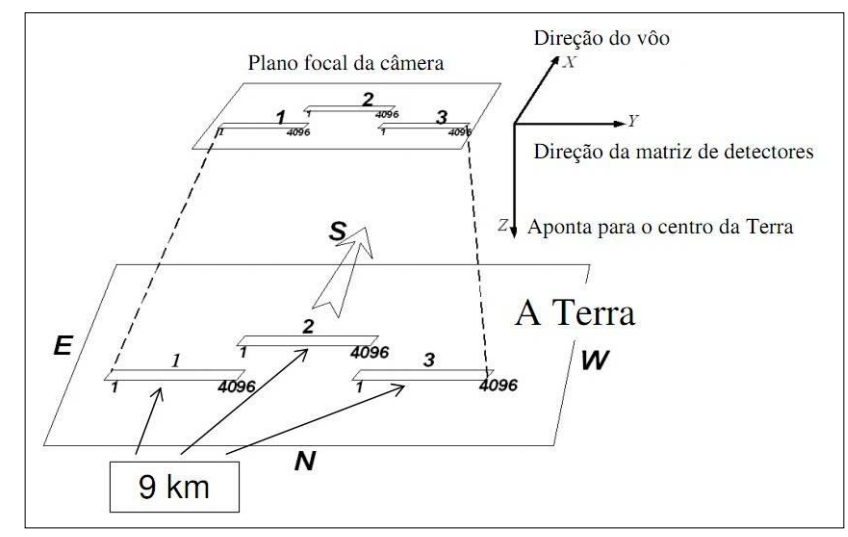

Figura 3: Plano focal do HRC.

Fonte: Epiphanio (2009).

O desalinhamento da matriz 2 em relação às demais pode ser considerado como um erro sistemático no plano focal do sensor, uma vez que há uma diferença no instante de aquisição para linhas correspondentes. Este desalinhamento, se não considerado na modelagem matemática, faz com que haja deslocamentos sistemáticos significativos nas coordenadas dos pontos no espaço objeto, na direção da trajetória do satélite. Tal fato foi constatado por Yue et al. (2009), Marcato Júnior (2011), Zhang e Zheng (2012) e Rodrigues e Ferreira (2013). Ainda de acordo com Zhang e Zheng (2012), a construção interna do sensor HRC é o que dificulta a correção geométrica destas imagens, uma vez que elas não podem ser tratadas como imagens lineares convencionais. Algumas vezes é possível identificar falhas na junção das imagens obtidas pelas matrizes, conforme ilustrado na Figura 4.

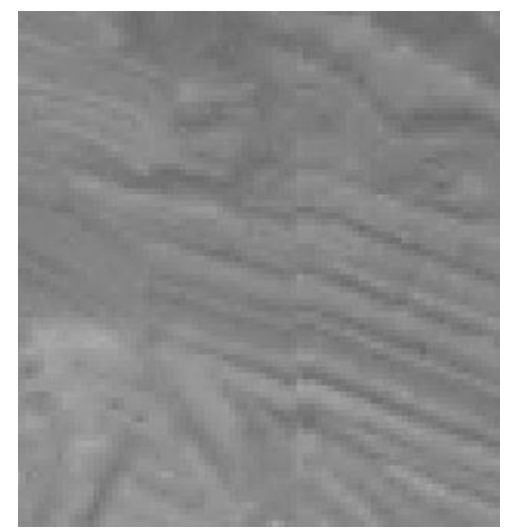

Figura 4: Erro na junção das imagens obtidas pelas matrizes CCD da esquerda e do centro.

Fonte: Zhang e Zheng (2012). 


\section{Modelo UCL para orientação exterior de imagens de varredura linear orbitais}

A orientação de imagens geralmente é realizada com o uso do modelo matemático rigoroso baseado nas Equações de Colinearidade. O princípio da colinearidade considera que o ponto no espaço imagem, o respectivo ponto no espaço objeto e o CP são colineares no instante de tomada da imagem. No caso de sensores de varredura linear as equações de colinearidade devem ser modificadas de forma a considerar a geometria de deslocamento do sensor, ou seja, as mudanças dos POEs no tempo. Como nos sensores de varredura linear cada linha corresponde a uma imagem diferente, a componente $x_{c}$ do SRC apresenta intervalo de zero ao valor do tamanho do pixel no CCD (Figura 1b). A Equação de Colinearidade modificada para sensores de varredura linear é apresentada nas Equações 4 e 5:

$$
\begin{aligned}
& x_{\mathrm{c}}=-f \frac{r_{11}(t)\left[X_{i}-X_{S}(t)\right]+r_{12}(t)\left[Y_{i}-Y_{S}(t)\right]+r_{13}(t)\left[Z_{i}-Z_{S}(t)\right]}{r_{31}(t)\left[X_{i}-X_{S}(t)\right]+r_{32}(t)\left[Y_{i}-Y_{S}(t)\right]+r_{33}(t)\left[z_{i}-Z_{S}(t)\right]}=-f \frac{\Delta X(t)}{\Delta Z(t)}, \\
& y_{c}=-f \frac{r_{21}(t)\left[X_{i}-X_{S}(t)\right]+r_{22}(t)\left[Y_{i}-Y_{S}(t)\right]+r_{23}(t)\left[z_{i}-Z_{S}(t)\right]}{r_{31}(t)\left[X_{i}-X_{S}(t)\right]+r_{32}(t)\left[Y_{i}-Y_{S}(t)\right]+r_{33}(t)\left[Z_{i}-Z_{S}(t)\right]}=-f \frac{\Delta Y(t)}{\Delta Z(t)},
\end{aligned}
$$

onde $X_{i}, Y_{i}$ e $Z_{i}$ são coordenadas do espaço objeto; $X_{S}(t), Y_{S}(t)$ e $Z_{S}(t)$ são as coordenadas do CP do sensor no tempo $t$ no Sistema de Referência Terrestre (SRT); $f$ é a distância focal calibrada do sensor e; $r_{11}(t), \ldots, r_{33}(t)$ são os componentes da matriz de rotação $R(t)$, responsável por alinhar o SRC com o SRT.

Mesmo considerando o princípio de colinearidade, de uma forma geral, os modelos rigorosos podem ser separados em duas classes, a saber: modelos Posição-Rotação e modelos ÓrbitaAtitude (Kim e Dowman, 2006).

Nos modelos Órbita-Atitude, para o alinhamento do sistema de referência do espaço imagem com o sistema de referência do espaço objeto são considerados dois sistemas de referência intermediários. Estes são: o Sistema de Referência da Plataforma (SRP) e o Sistema de Referência Orbital (SRO). Deste modo, são utilizados na modelagem o SRC, o SRP, o SRO e o SRT. A transformação do SRC para o SRP é feita a partir dos dados de translação do CP do sensor para o centro de massa do satélite e de uma matriz de rotações em função dos ângulos de visada do sensor (boresight). Para transformar do SRP para o SRO utiliza-se uma matriz de rotação, proveniente da multiplicação de três matrizes de rotação em função dos ângulos de atitude da plataforma rolamento $(R)$, arfagem $(P)$ e guinada $(\psi)$. Por fim, para a transformação do SRO para o SRT tem-se uma matriz de rotação em função das componentes de posição e velocidade do satélite no instante de formação da imagem. Considerando a estrutura desta modelagem, os dados de órbita disponibilizados em arquivos auxiliares das imagens, como posição, velocidade e ângulos de atitude podem ser utilizados diretamente na orientação. Mais detalhes sobre o equacionamento e aplicações deste modelo podem ser vistos em Radhadevi et al. (1994) e Kim e Dowman (2006). Aplicações com as imagens CCD e HRC do satélite CBERS 2B podem ser encontradas em Marcato Júnior et al. (2011) e Marcato Júnior e Tommaselli (2013).

Já no caso dos modelos Posição-Rotação, o alinhamento do sistema de referencia do espaço imagem com o do espaço objeto considera apenas o SRC e o SRT. Deste modo, apenas uma matriz de rotações em função de ângulos de orientação denominados ômega, phi e kappa $(\omega, \varphi$, $\kappa)$. Esta configuração impede que os dados de atitude $(R, P, \psi)$ disponibilizados nos metadados das imagens possam ser utilizados diretamente. Esta classe de modelo é a mais utilizada nos aplicativos comerciais atualmente. 
Considerando a estrutura dos modelos Posição-Rotação, diversas abordagens distintas foram desenvolvidas para a modelagem das variações dos POEs durante a formação da cena. O modelo desenvolvido por Gugan e Dowman (1988) apresenta o relacionamento entre os parâmetros de orientação exterior e o tempo $t$ modelado por polinômios de segunda ordem. Orun e Natarajan (1994) propuseram um conjunto reduzido de parâmetros polinomiais, indicando que devido à própria característica física da aquisição de cenas, pequenas variações em $\omega$ tem o mesmo efeito que pequenas variações em $Y_{s}$ e; pequenas variações em $\varphi$ assemelham-se muito à pequenas variações em $X_{s}$. Assim, no modelo de plataforma final, os autores propuseram a injunção absoluta dos ângulos $\omega$ e $\varphi$. Além disso, indicaram que, uma vez que a estimativa de tempo $(t)$ é função linear da linha da imagem $(t=d t \cdot L)$, pode-se admitir que $L$ substitui a variação de tempo $t$, e as mudanças em unidades (fator de escala entre $t$ e $L$ ) podem ser absorvidas pelos coeficientes polinomiais.

Partindo-se da ideia que a propagação da posição do satélite no modelo de plataforma pode ser determinada a partir de um polinômio de segunda ordem, Michalis e Dowman (2005) desenvolveram um modelo de plataforma denominado primeiramente pelos autores de Modelo de Kepler, vindo a ser denominado posteriormente de Modelo UCL (University College of London), conforme pode ser visto em Michalis e Dowman (2010) e Dowman et al. (2011). Neste modelo, os coeficientes lineares das componentes da variação da posição são substituídos pela velocidade da plataforma e os termos quadráticos pelas acelerações. A partir destas considerações, as acelerações utilizadas nos polinômios de segundo grau podem ser calculadas a partir da Equação do Movimento Kepleriano (Seeber, 2003) e as equações do modelo da plataforma, associadas à teoria do Movimento uniformemente variado (MUV):

$$
\begin{gathered}
X_{S}(t)=X_{0}+u_{x} \tau-\frac{G M \cdot X_{0} \cdot \tau^{2}}{2 \cdot\left(X_{0}{ }^{2}+Y_{0}{ }^{2}+Z_{0}{ }^{2}\right)^{3 / 2}}, \\
Y_{S}(t)=Y_{0}+u_{y} \tau-\frac{G M \cdot Y_{0} \cdot \tau^{2}}{2 \cdot\left(X_{0}{ }^{2}+Y_{0}{ }^{2}+Z_{0}{ }^{2}\right)^{3 / 2}}, \\
Z_{S}(t)=Z_{0}+u_{z} \tau-\frac{G M \cdot Z_{0} \cdot \tau^{2}}{2 \cdot\left(X_{0}{ }^{2}+Y_{0}{ }^{2}+Z_{0}{ }^{2}\right)^{3 / 2}}, \\
\omega=\omega_{0}, \\
\varphi=\varphi_{0}, \\
\kappa=\kappa_{0},
\end{gathered}
$$

onde $X_{0}, Y_{0}, Z_{0}$ são as componentes do vetor posição na primeira linha da imagem, $u_{x}, u_{y}$, $u_{z}$ são as componentes do vetor velocidade na primeira linha da imagem; $\tau$ é o produto entre $L$ e o tempo de integração da linha CCD do sensor $(d t)$ e; $G M$ é a constante gravitacional geocêntrica (particular para cada Sistema de Referência Terrestre).

Originalmente, em Michalis e Dowman (2005), nota-se que os ângulos de atitude são considerados invariáveis durante o processo de formação das imagens. Porém, esta configuração pode ser alterada mediante o modo de aquisição de imagens de cada satélite. Um exemplo de alteração para cenas PRISM-ALOS pode ser visto em Dowman et al. (2011).

Para a utilização deste modelo de plataforma, a orientação das imagens deve ser realizada em um Sistema de coordenadas geocêntrico, a fim de evitar distorções causadas pela curvatura da Terra e da projeção cartográfica e, ainda, para facilitar a integração com as informações dos metadados (Michalis e Dowman, 2008a). Nesse caso, é ainda considerado que as linhas que compõem a imagem são adquiridas com um intervalo de tempo constante. Como resultado as coordenadas ao longo da trajetória da plataforma têm a mesma escala.

Uma importante consideração neste modelo é que o movimento do satélite durante o tempo de aquisição de imagens estéreo along track é considerado um movimento Kepleriano. Por resta razão, as coordenadas do espaço objeto de pontos de controle ou verificação devem estar referenciadas ao Sistema de Referência Celeste Geocêntrico (SRCG). Desta forma, como um primeiro passo antes da orientação, as coordenadas, tanto dos pontos de controle como os de 
verificação, coletadas originalmente no SRT, devem ser transformadas para o SRCG. Com o objetivo de suprimir tal procedimento, Rodrigues et al. (2013) adaptaram o Modelo UCL para o uso do Problema dos Dois Corpos no SRT, inserindo termos em função da velocidade angular de rotação da Terra. Tendo em vista que o intervalo de obtenção das cenas HRC-CBERS 2B é relativamente pequeno ( 4s), a desconsideração das componentes de Precessão, Nutação e Movimento do Polo não causam prejuízo significativo. Mais detalhes sobre o equacionamento podem ser vistos em Leick (2004).

Exemplos de aplicação deste modelo podem ser vistos em Dowman e Michalis (2003) utilizando cenas ASTER; Michalis e Dowman (2004), Michalis (2005), Michalis e Dowman (2006), Michalis e Dowman (2008a) com cenas HRS-SPOT 5; Michalis e Dowman (2008b) com cenas do satélite CARTOSAT-I; Dowman et al. (2011), Michalis e Dowman (2010), Rodrigues et al. (2013) em cenas PRISM-ALOS e; Rodrigues e Ferreira (2013), Machado et al. (2013) utilizando cenas HRC-CBERS 2B.

\section{Calibração geométrica em órbita de sensores pushbroom lineares}

Conforme visto anteriormente, para a orientação de imagens orbitais é necessário o conhecimento de alguns POIs como a distância focal, o tamanho do pixel na matriz de CCD e o número de colunas da imagem. No entanto, quando se requer resultados ao nível sub-pixel na orientação, é imprescindível que se leve em consideração também os parâmetros relacionados com as características geométricas do plano focal do sensor e com as imperfeições de fabricação e montagem do mesmo.

$\mathrm{Na}$ grande maioria dos sensores orbitais, o arranjo das matrizes de CCD no plano focal é do tipo de constituição em linha, no qual uma ou mais matrizes formam uma única linha da imagem (Poli, 2005). No caso de múltiplas matrizes, estas são colocadas uma ao lado da outra, ao longo de uma única linha, conectadas por uma pequena sobreposição, conforme mencionado anteriormente. Sensores como os dos satélites Orbview-3, IKONOS-2, QUICKBIRD-2, PRISMALOS e HRC-CBERS 2B são exemplos de sensores com múltiplas matrizes de arranjo de constituição em linha. Em teoria, as matrizes de CCD devem ser exatamente alinhadas ou no mínimo paralelas, sendo as posições das mesmas em relação ao PP conhecidas no plano focal (Jacobsen, 2005). Porém, durante a fabricação imperfeições podem ocorrer, gerando pequenos deslocamentos bidirecionais, rotações, alterações de tamanho e afastamento de linearidade (flexão) nas matrizes.

As características geométricas e as imperfeições de fabricação citadas constituem-se em erros sistemáticos. Parâmetros relacionados a estes erros devem fazer parte do conjunto de POIs do sensor, podendo ser estimados antes do lançamento do satélite, a partir de uma calibração em laboratório. Porém, mesmo que haja este procedimento, uma reestimativa dos valores após o satélite ser colocado em órbita também deve ocorrer, ou seja, uma calibração geométrica em órbita.

Segundo Batlsavias et al. (2006), a calibração geométrica em órbita é importante para sensores orbitais, uma vez que durante o lançamento do satélite as condições ambientais se alteram de forma rápida e drástica, causando mudanças na geometria interna do sensor. Segundo os mesmos autores, mudanças geométricas não tão cruciais como as do caso anterior, mas ainda prejudiciais, também podem ocorrer devido às condições ambientais duras impostas ao satélite, após a estabilização do mesmo em órbita. Deste modo, os autores destacam a importância da realização frequente da calibração geométrica em órbita pelas agências que operam os satélites. Ainda de 
acordo com Jacobsen (2005), as acelerações impostas durante o lançamento do satélite podem causar mudanças nas posições exatas das matrizes de CCD, o afastamento da linearidade geométrica (flexões) das mesmas e alterar as condições iniciais do sistema de lentes. $\mathrm{O}$ autor indica ainda, que a influência termal do sol quando o satélite encontra-se em órbita pode também causar mudanças geométricas, sendo recomendada a verificação dos valores calibrados de tempos em tempos.

A calibração geométrica em órbita é um ajustamento simultâneo por feixes de raios perspectivos com parâmetros adicionais nas Equações de Colinearidade modificadas, referentes aos POIs a serem reestimados. Nesta técnica utiliza-se uma área de teste, com um conjunto denso, preciso e bem distribuído de pontos de controle no terreno sobre toda a imagem (Baltsavias et al., 2006). O principal interesse na aplicação desta técnica é a reconstrução acurada de feições do espaço objeto simultaneamente com a estimativa dos POIs que recuperam a condição da colinearidade no instante da formação da imagem.

Poli (2005), ao considerar diferentes POIs a serem estimados, propôs uma separação para os mesmos, indicando os relacionados com o sistema óptico e os relacionados com as matrizes de CCD. Os POIs relacionados ao sistema óptico compreendem o deslocamento do PP, a variação da distância focal $(\Delta f)$, os parâmetros de distorção radial simétrica e descentrada das lentes $\left(k_{1}, k_{2}\right.$ e $\left.P_{1}, P_{2}\right)$ e a variação de escala. Já os POIs relacionados com as matrizes CCD são a mudança na dimensão do pixel $(s)$, deslocamentos $(\Delta x$ e $\Delta y)$ ou rotações $(\theta)$ das matrizes de CCD no plano focal com relação às suas posições nominais e o ângulo central do efeito da flexão (não linearidade geométrica - $b$ ). O efeito dos POIs relacionados com as matrizes CCD no plano focal podem ser vistos na ilustração da Figura 5. Os equacionamentos dos POIs como parâmetros adicionais nas Equações de Colinearidade podem ser vistos detalhadamente em Poli (2005) e em Kocaman (2007).

Exemplos de calibrações em órbita podem ser vistos em Jacobsen (1997) com imagens IRS-1C PAN, Kornus et al. (1998) com imagens MOMS-2P, Mulawa (2003) em imagens Orbview-3, Tadono et al. (2004) com imagens PRISM-ALOS, Gachet (2004) para imagens HRG e HRSSPOT 5, Poli (2005) com imagens MOMS-P2, HRS-SPOT 5, ASTER, MISR e EROS-Al, Baltsavias et al. (2006) em imagens IKONOS-2, Toutin et al. (2006) com imagens HRS/HRGSPOT 5 e RSI-FORMOSAT-2, Gruen et al. (2007) e Kocaman (2007) para imagens PRISMALOS, Radhadevi e Solanki (2008) com imagens do satélite IRS P6 e Wang et al. (2014) com imagens da câmera pancromática do satélite ZY1-02C e imagens do satélite ZY-3. Cabe ressaltar que em todos os trabalhos citados as calibrações foram realizadas com imagens singulares ou estéreo imagens convergentes obtidas na mesma órbita (along track).

Especificamente para as imagens do sensor HRC, Yue et al. (2009) adicionou nas Equações de Colinearidade três parâmetros de translação de matrizes de CCD ao longo da órbita (eixo $x_{c}$ ) referentes às três matrizes presentes no plano focal. Entretanto, os erros sistemáticos decorrentes dos deslocamentos das matrizes de CCD na direção across-track (eixo $y_{c}$ ) ainda permaneceram. Além disso, distorções provenientes da imperfeição do sistema de lentes e rotações nas matrizes em relação às condições nominais não foram modeladas. 


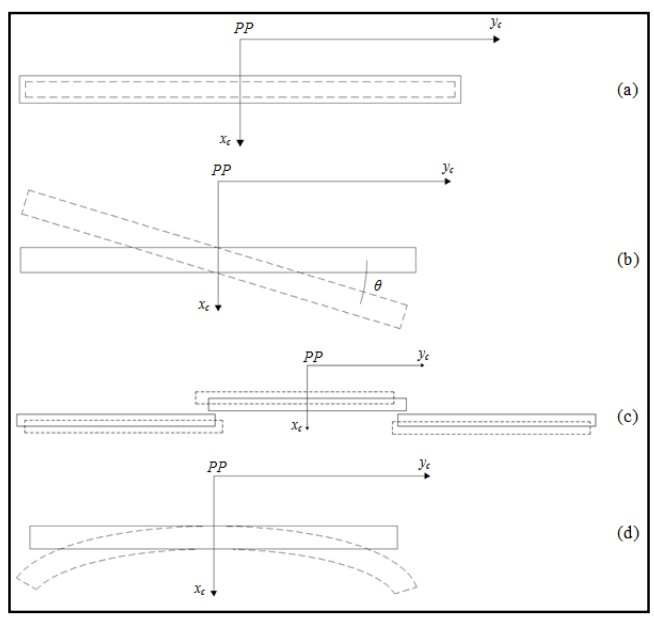

Figura 5: Efeito dos POIs relacionados com as matrizes CCD no plano focal: (a) mudança na dimensão do pixel, (b) rotação da matriz de CCD, (c) deslocamentos das matrizes de CCD e (d)

Flexão na matriz de CCD.

Zhang e Zheng (2012) analisaram a consideração de diferentes grupos de POIs, sendo que o mais completo foi aquele contendo 2 parâmetros de translação e 1 de rotação para cada matriz de CCD, além da distância focal. Os resultados mostraram uma melhora de 2 a 3 pixels nos resultados finais a partir da análise de pontos de verificação. No entanto, os autores indicaram que devido à pequena influência nos resultados finais e à baixa qualidade dos pontos de controle e verificação, extraídos por procedimento automático de cartas topográficas 1:10.000, as alterações no tamanho do pixel e os afastamentos de linearidade das matrizes de CCD (flexões) não foram considerados. Além disso, as distorções do sistema de lentes também foram desconsideradas no ajustamento. Cabe ressaltar que nenhum valor de POI obtido por calibração geométrica, tanto do plano focal como do sistema de lentes em laboratório foi divulgado nos metadados das imagens HRC distribuídas.

\section{Metodologia}

\section{1 Área de Teste}

A imagem utilizada como área de teste possui nível de processamento 1, com as observações de posição e velocidade do satélite disponibilizadas no metadado referenciadas ao STR WGS-84 (G1150). Para a extração das componentes altimétricas dos pontos de verificação e controle foram utilizados Modelos Digitais de Elevação (MDE) ASTER-GDEM referenciados ao mesmo STR. A área, recoberta pela imagem HRC-CBERS 2B, órbita-ponto 157_E_120_5 de 14/09/2009, abrange parte da região sudeste do estado de Goiás e sudoeste do estado de Minas Gerais. Esta área encontra-se em região de planalto com pouca variação de altitude, sendo o maior desnível extraído do MDE para a área da imagem $(27$ x $27 \mathrm{~km})$ igual a $324 \mathrm{~m}$. 
Nesta área de teste foram coletados 63 pontos de controle e 36 pontos de verificação, todos obtidos a partir de ortoimagens com resolução espacial, ou seja, GSD de $1 \mathrm{~m}$ cedidas pelo Instituto Brasileiro de Geografia e Estatística (IBGE). Tanto os pontos de controle como os de verificação tiveram as coordenadas do SRI coletadas de forma manual. O desvio-padrão para as coordenadas da imagem, foi considerado igual a 3 pixels $(0,030 \mathrm{~mm})$. A distribuição dos pontos sobre as imagens pode ser visualizada na Figura 6. Devido à necessidade de se ter uma distribuição densa de pontos de controle para atender ao processo de calibração geométrica, houve uma dificuldade de se selecionar locais adequados para os pontos de verificação, isto é, com significativa distância dos pontos de controle. Porém, mesmo com essa dificuldade, procurou-se selecionar os pontos de verificação em locais mais distantes possíveis dos pontos de controle.

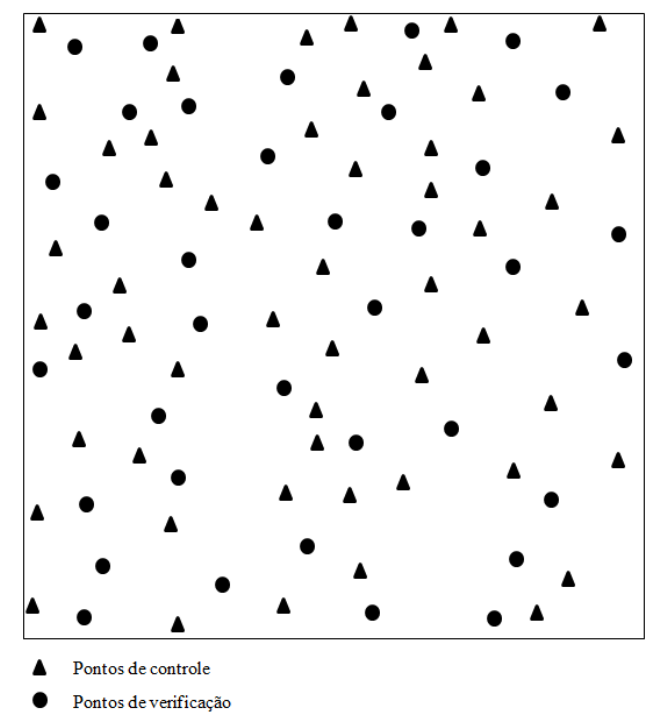

Figura 6: Distribuição dos pontos de controle e verificação na imagem.

\subsection{Preparação dos dados}

Após a coleta de pontos, tanto os de controle quanto os de verificação, houve a separação de pontos por matrizes de CCD. Tal procedimento foi necessário uma vez que quando considerada a calibração geométrica em órbita as orientações foram teoricamente realizadas em cada matriz separadamente. Ao ter em vista que todas as três matrizes possuem o mesmo tamanho, ou seja, 4082 colunas, a separação foi feita com base apenas nos valores de coordenadas de coluna do SCI, a saber: matriz 1 de 0 a 4082, matriz 2 de 4083 a 8164 e matriz 3 de 8165 a 12246 . A divisão dos pontos para as matrizes é apresentada na Figura 7. 


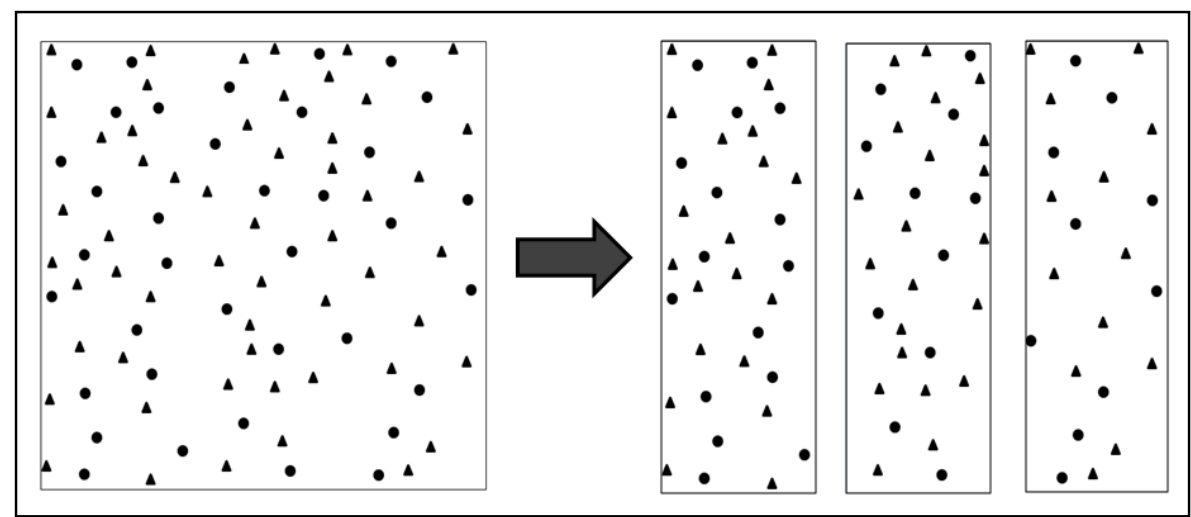

Figura 7: Distribuição dos pontos de controle e verificação nas matrizes de CCD.

Uma vez que a imagem possui um arquivo de metadado no formato $x \mathrm{ml}$ contendo as efemérides do satélite referentes aos instantes adjacentes ao instante de início do imageamento, foi possível obter, via interpolação, os vetores de estado neste instante. $\mathrm{O}$ interpolador utilizado foi a spline cúbica, adotando para o conjunto de dados de interpolação 10 valores em torno do instante de cálculo, com intervalo de 1 segundo, conforme disponibilizado nos arquivos.

As efemérides do CBERS 2B contidas no arquivo metadado podem ter duas fontes, a saber: o receptor GPS embarcado ou as já bem difundidas efemérides TLE (Two Line Elements) da NASA. No caso das imagens utilizadas nesta pesquisa a segunda fonte foi verificada, não estando disponíveis as efemérides obtidas por GPS. Um exemplo de dados de efemérides contidos no arquivo .xml é apresentado na Figura 8. $\mathrm{O}$ vetor de estado referente à primeira linha da imagem é apresentado na Tabela 2.

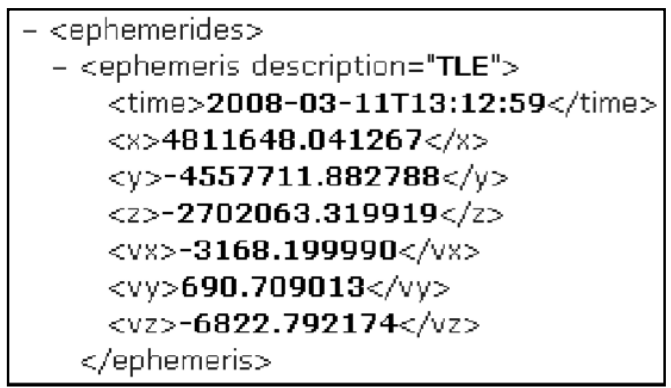

Figura 8: Efemérides disponibilizadas no arquivo de metadados.

Tabela 2: Vetor de estado interpolado para a imagem.

\begin{tabular}{c|c}
\hline & Imagem 3 \\
\hline $\mathbf{X ~ ( m )}$ & 4588775,564 \\
\hline $\mathbf{V x ~ ( m / s )}$ & $-2719,047$ \\
\hline $\mathbf{Y ~ ( m )}$ & $-5037255,894$ \\
\hline $\mathbf{V y ~ ( m / s )}$ & 532,640 \\
\hline $\mathrm{Z} \mathrm{(m)}$ & $-2183027,096$ \\
\hline $\mathbf{V z}(\mathbf{m} / \mathbf{s})$ & $-7025,376$ \\
\hline
\end{tabular}

\subsection{Modelagem matemática}


Para considerar as características da geometria interna do sensor HRC-CBERS 2B, as Equações de Colinearidade devem conter os POIs como parâmetros adicionais. Nesta pesquisa foram considerados como POIs os deslocamentos de cada centro geométrico das matrizes de CCD da esquerda, do centro e da direita, em relação ao PP; rotações das matrizes de CCD em relação ao eixo $y_{c}$; o fator de escala no eixo $y_{c}$ em cada matriz de CCD; a flexão em forma de arco de cada matriz de CCD e; os parâmetros $k_{1}$ e $k_{2}$ de distorção radial do sistema de lentes. Cabe ressaltar que os parâmetros de distorção descentrada do sistema de lentes $P_{1}$ e $P_{2}$ não foram considerados por não existir a possibilidade de diferenciá-los de $k_{1}$ e $k_{2}$, uma vez que não existe possibilidade de se ter uma imagem com um giro de $90^{\circ}$ em torno do eixo óptico da câmara com relação à faixa de trajetória do satélite (Andrade, 2003). Outro POI desconsiderado foi o parâmetro $\Delta f$, referente à variação da distância focal, uma vez que o efeito sistemático proveniente da desconsideração deste POI pode ser absorvido pelo parâmetro $Z_{0}$ do conjunto de POEs. Considerando todos os POIs citados nas coordenadas $x_{c}$ e $y_{c}$ do SRC, tem-se as Equações 7 e 8:

$$
\begin{gathered}
x_{c i}=x_{s i}+\Delta x_{i}+y_{s i} \operatorname{sen} \theta_{i}+\delta x_{b_{i}}+\delta x_{R_{i}}, \\
y_{c i}=y_{s i}+\Delta y_{i}-y_{s i} s_{i}+y_{s i}\left(1-\cos \theta_{i}\right)+\delta y_{R_{i}},
\end{gathered}
$$

sendo $i=\mathrm{E}, \mathrm{M}, \mathrm{D}$ indicando as matrizes de $\mathrm{CCD}$ da esquerda, central e da direita respectivamente; $x_{c i}$ e $y_{c i}$ as coordenadas do SRC corrigidas; $\Delta x_{i}, \Delta y_{i}$ os deslocamentos do centro geométrico da matriz de CCD $i$ em relação ao PP; $\theta i$ a rotação da matriz de CCD $i$ em relação ao eixo $y_{c} ; s_{i}$ o fator de escala da matriz de CCD $i$ na direção do eixo $y_{c} ; \delta x_{b i}$ a correção do efeito de flexão da matriz de CCD i e; $\delta x_{R i}$ e $\delta y_{R i}$ as correções da distorção radial simétrica do conjunto de lentes. A correção do efeito de flexão para uma matriz de CCD $i$ é dada pela Equação 9 (Poli, 2005):

$$
\delta x_{b_{i}}=-\left(\frac{n \operatorname{col} l_{i} P S}{2 \operatorname{sen} \frac{b_{i}}{2}}\right)\left[\cos \left(\frac{y_{s i} 2 \operatorname{sen} \frac{b_{i}}{2}}{n \operatorname{col} l_{i} P S}\right)-\cos \frac{b_{i}}{2}\right],
$$

onde col $_{i}$ é o número de colunas da matriz de CCD $i$; PS o tamanho do pixel na matriz de CCD $i$ (igual para as três) e; $b_{i}$ o ângulo de flexão da matriz de CCD $i$. Outro tipo de equacionamento para este erro sistemático pode ser visto em Kocaman (2007). Para os termos $\delta x_{R i}$ e $\delta y_{R i}$ referentes às correções das distorções radiais do sistema de lentes foi utilizado o modelo de Brown expresso pelas Equações 10 a 15 (1971):

$$
\begin{gathered}
\delta x_{R_{E}}=\left[L_{E}-\operatorname{Int}\left(L_{E}\right)\right] P S\left(r_{E}^{2} k_{1}+r_{E}^{4} k_{2}\right), \\
\delta y_{R_{E}}=-\left[\frac{n \operatorname{col}_{M}}{2}+\left(n \operatorname{col}_{E}-C_{E}\right)\right] P S\left(r_{E}^{2} k_{1}+r_{E}^{4} k_{2}\right), \\
\delta x_{R_{M}}=\left[L_{M}-\operatorname{Int}\left(L_{M}\right)\right] P S\left(r_{M}^{2} k_{1}+r_{M}^{4} k_{2}\right), \\
\delta y_{R_{M}}=\left[-\frac{n c o l_{M}}{2}+\left(C_{M}-n \operatorname{col}_{E}\right)\right] P S\left(r_{M}^{2} k_{1}+r_{M}^{4} k_{2}\right), \\
\delta x_{R_{D}}=\left[L_{D}-\operatorname{Int}\left(L_{D}\right)\right] P S\left(r_{D}^{2} k_{1}+r_{D}^{4} k_{2}\right), \\
\delta y_{R_{D}}=\left[\frac{n \operatorname{cool}_{M}}{2}+\left(C_{D}-n \operatorname{col}_{E}-n \operatorname{col}_{M}\right)\right] P S\left(r_{D}^{2} k_{1}+r_{D}^{4} k_{2}\right) .
\end{gathered}
$$

onde $L_{E}, C_{E}, L_{M}, C_{M}, L_{D}, C_{D}$ são as coordenadas do SRI separadamente para as matrizes da esquerda, central e direita, respectivamente e; $n \operatorname{col}_{E}, n \operatorname{col}_{M}, n \operatorname{col}_{D}$ são os números de colunas das matrizes de CCD da esquerda, centro e direita, respectivamente. As Equações de Colinearidade com os POIs adicionados, para a matriz de CCD $i$ são as Equações 16 e 17: 


$$
\begin{aligned}
& x_{s i}=-f \frac{\Delta x_{i}}{\Delta z_{i}}-\Delta x_{i}-y_{s i} \operatorname{sen} \theta_{i}-\delta x_{b_{i}}-\delta x_{R_{i}}, \\
& y_{s i}=-\frac{f \Delta Y_{i}}{\Delta z_{i}}-\Delta y_{i}+y_{s i} s_{i}-y_{s i}\left(1-\cos \theta_{i}\right)-\delta y_{R_{i}},
\end{aligned}
$$

onde $\Delta X_{i}$ é a parte superior da razão das Equações de Colinearidade na Equação (4) para a matriz de CCD $i ; \Delta Y_{i}$ é a parte superior da razão das Equações de Colinearidade na Equação (5) para a matriz de CCD $i$ e; $\Delta Z i$ é a parte inferior da razão das Equações de Colinearidade nas Equações (4) e (5) para a matriz de CCD $i$.

O modelo de plataforma utilizado nesta pesquisa é o Modelo UCL com a modificação do uso de coordenadas do SRT ao invés de coordenadas do SRCG, conforme apresentado em Rodrigues et al. (2013). Neste modelo, o intervalo de tempo de aquisição das linhas CCD das imagens foi considerado constante. Uma vez conhecidos os vetores de estado do tempo inicial de formação das imagens, estes receberam injunção relativa de $3 \mathrm{~km}$ e $1 \mathrm{~km} / \mathrm{s}$ respectivamente para as componentes da posição e da velocidade. As efemérides TLE referem-se ao centro de massa do satélite $(\mathrm{CM})$, porém, para realizar a orientação, as posições devem referir-se ao CP da câmera HRC. Neste caso, como estas quantidades são de ordem métrica, os deslocamentos entre o CM e o CP do sensor foram agrupados nos desvios padrão associados na pesagem das injunções relativas em cada imagem. Ainda com relação ao modelo de plataforma, os ângulos $\omega$ e $\varphi$ foram estimados nos ajustamentos sem injunções absolutas, uma vez que foi utilizado o Sistema de Coordenadas Geocêntrico Cartesiano Terrestre, o que leva a não incidência de correlações altas com $Y_{S}$ e $X_{S}$ (Marcato Júnior, 2011). O ângulo $\kappa$ foi considerado variável e propagado a partir de polinômios de $2^{\circ}$ grau, tendo em vista o movimento caranguejo do CBERS $2 \mathrm{~B}$ citado anteriormente. Tem-se então as Equações 18:

$$
\begin{gathered}
X_{s}(t)=X_{0}+u_{x} t+\left[-\frac{G M \cdot X_{0}}{2 \cdot r^{3}}+\omega_{t}{ }^{2} X_{0}+2 \Omega_{t} u_{y}\right] \cdot t^{2}, \\
Y_{s}(t)=Y_{0}+u_{y} t+\left[-\frac{G M \cdot Y_{0}}{2 \cdot r^{3}}+\omega_{t}{ }^{2} Y_{0}+2 \Omega_{t} u_{x}\right] \cdot t^{2}, \\
Z_{s}(t)=Z_{0}+u_{z} t-\frac{G M \cdot Z_{0}}{2 \cdot r^{3}} \cdot t^{2}, \\
\omega=\omega_{0}, \\
\varphi=\varphi_{0}, \\
\kappa=\kappa_{0}+d_{1} t+d_{2} t^{2},
\end{gathered}
$$

onde:

$$
r=\sqrt{X_{0}^{2}+Y_{0}^{2}+Z_{0}^{2}}
$$

sendo $d_{1}$ e $d_{2}$ os coeficientes polinomiais da variação do ângulo $\kappa_{0}, \Omega_{\mathrm{t}}$ a velocidade angular de rotação da Terra assumindo o valor de 7292115 x 10-11 rad s-1; e GM = 3986005 x 108 m3s2; ambos referentes aos valores adotados pelo STR WGS 84 G1150, no qual os vetores de estado estão referenciados.

Um ponto importante que cabe ser ressaltado é que conforme apresentado no item 3 , a imagem final em nível de processamento 1, com 12246 colunas, é formada a partir de um processo geométrico com base em algoritmos de correlação que realizam a fusão das sub-imagens provenientes das três matrizes de CCD. Partindo do princípio que a área imageada pela matriz central sempre estará à frente das áreas imageadas pelas matrizes laterais, quando ocorre o alinhamento das sub-imagens para a montagem da imagem final deve-se considerar que o instante do imageamento da área da matriz central é anterior ao instante de imageamento das áreas das matrizes laterais. Neste caso, deve haver uma alteração na quantidade $t$ nos modelos de plataforma utilizados para as orientações das sub-imagens laterais, somando-se o afastamento em linhas das sub-imagens laterais para a sub-imagem central, que conforme pode ser visto na Figura 3 é de $26 \mathrm{~mm}$ ou 2600 pixels. A quantidade $t$ para os modelos de plataforma das subimagens laterais é dada pela Equação 20: 


$$
t=d t \cdot(L+2600) .
$$

É importante ressaltar que se este procedimento não for realizado, os POIs não apresentarão significado físico, como no caso dos POIs estimados por Zhang e Zheng (2012) e Yue et al. (2009).

Com relação aos ajustamentos de observações em todos os experimentos, foi utilizado o Método Paramétrico, tendo em vista que as observações foram colocadas de forma explícita com relação aos parâmetros. Os POIs referentes à matriz central, ou seja, $\Delta x_{c}, \Delta y_{c}, \theta_{c}$ e $s_{c}$ receberam injunções absolutas, com valores iguais a zero. O procedimento de tornar uma das matrizes fixa no ajustamento é necessário para poder estabelecer o referencial da câmera e evitar singularidades, conforme pode ser visto em Poli (2005), Weser et al. (2008) e Zhang e Zheng (2012). Associando estes valores, assume-se que o PP é coincidente com o centro geométrico da matriz central e que esta não possui rotação em relação ao eixo y do SRC. Os erros sistemáticos decorrentes deste procedimento são assumidos pelos POEs, conforme indicado por Weser et al. (2008). Com relação ao fator de escala injuncionado como 1, assume-se que a matriz de CCD não sofreu nenhuma dilatação ou contração sobre o seu comprimento nominal. A parte dos POEs referentes ao vetor de estado da plataforma $\left(X_{0}, Y_{0}, Z_{0}, u_{x}, u_{y}, u_{z}\right)$ receberam injunções relativas de peso com pesos inversamente proporcionais ao quadrado das precisões, adotadas como 3000 $\mathrm{m}$ e $100 \mathrm{~m} / \mathrm{s}$ para as componentes da posição e velocidade respectivamente.

\subsection{Experimentos}

A imagem tomada com área de teste foi utilizada para a realização de quatro experimentos com o uso de dois diferentes grupos de POIs. O primeiro experimento foi realizado sem uso dos POIs, considerando apenas uma única matriz de CCD com 12246 colunas, sendo fruto da fusão das três matrizes da forma como as imagens em nível 1 são disponibilizadas. No segundo experimento foi utilizado um primeiro grupo de POIs contendo os parâmetros de translação e rotação das matrizes de $\mathrm{CCD}$ em relação à matriz de CCD central. Neste caso, utilizam-se as Equações de (22) a (27) sem os POIs relacionados com os fatores de escala, flexão e distorção radial das lentes. No terceiro experimento foi utilizado um segundo grupo de POIs, contendo todos do experimento 2, mais os fatores de escala na direção do eixo $y_{c}$ nas matrizes laterais e as flexões das três matrizes de CCD. No terceiro grupo de POIs, utilizado no experimento 4, foram considerados todos do segundo grupo de POIs adicionando-se a modelagem da distorção radial simétrica das lentes. A síntese dos parâmetros dos experimentos realizados de 1 a 4 pode ser verificada na Tabela 3. Cabe ressaltar que o grupo de POIs considerado no experimento 2 é o mesmo grupo de POIs do experimento 4 realizado em Zhang e Zheng (2012).

\section{Resultados}


Após as realizações das calibrações uma análise de determinabilidade dos POIs foi realizada. Para tanto, os valores dos POIs estimados nos ajustamentos, bem como suas precisões extraídas da matriz variância-covariância dos parâmetros ajustados foram analisados (Tabela 4).

Tabela 3: Parâmetros dos experimentos realizados de 1 a 4.

\begin{tabular}{|c|c|c|}
\hline & Característica & Parâmetros \\
\hline 1 & POE + Sem POIs. & $X_{0}, Y_{0}, Z_{0}, u_{x}, u_{y}, u_{z}, \omega, \varphi, \kappa, d_{1}, d_{2}$ \\
\hline 2 & $\mathrm{POE}+1^{\circ}$ grupo de POIs. & $\begin{array}{l}X_{0}, Y_{0}, Z_{0}, u_{x}, u_{y}, u_{z}, \omega, \varphi, \kappa, d_{1}, d_{2} \\
+\Delta x_{E}, \Delta y_{E}, \theta_{E^{\prime}}, \Delta x_{D}, \Delta y_{D}, \theta_{D}\end{array}$ \\
\hline 3 & $\mathrm{POE}+2^{\circ}$ grupo de POIs & $\begin{array}{l}X_{0}, Y_{0}, Z_{0^{\prime}}, u_{x^{\prime}} u_{y}, u_{z}, \omega, \varphi, \kappa, d_{1}, d_{2}, \\
+\Delta x_{E}, \Delta y_{E}, \theta_{E}, \Delta x_{D}, \Delta y_{D}, \theta_{D}, s_{E}, s_{D}, b_{E}, b_{C}, b_{D}\end{array}$ \\
\hline 4 & $\mathrm{POE}+3^{\circ}$ grupo de POIs. & $\begin{array}{l}X_{0}, Y_{0}, Z_{0}, u_{x}, u_{y}, u_{z}, \omega, \varphi, \kappa, d_{1}, d_{2}, \\
+\Delta x_{E}, \Delta y_{E}, \theta_{E}, \Delta x_{D}, \Delta y_{D}, \theta_{D}, s_{E}, s_{D}, b_{E}, b_{C}, b_{D}, k_{1}, k_{2}\end{array}$ \\
\hline
\end{tabular}

Tabela 4: POIs estimados e sua precisões nos experimentos 2,3 e 4.

\begin{tabular}{|c|c|c|c|c|c|c|}
\hline & \multicolumn{2}{|c|}{ Experimento 2} & \multicolumn{2}{|c|}{ Experimento 3} & \multicolumn{2}{|c|}{ Experimento 4} \\
\hline & POI & $\sigma_{\mathrm{POI}}$ & POI & $\sigma_{\mathrm{POI}}$ & POI & $\sigma_{\mathrm{POI}}$ \\
\hline$\Delta x_{E}$ & 25,8893 & 0,0201 & 25,8191 & 0,0365 & 25,8191 & 0,0366 \\
\hline$\Delta y_{E}$ & $-38,2768$ & 0,0174 & $-38,2488$ & 0,0227 & $-38,3791$ & 0,1171 \\
\hline$\theta_{E}$ & $-0,0034$ & 0,0006 & $-0,0028$ & 0,0007 & $-0,0028$ & 0,0007 \\
\hline$S_{E}$ & ---- & ---- & 0,0017 & 0,0006 & $-0,0046$ & 0,0058 \\
\hline$b_{E}$ & --- & --- & $-0,0002$ & 0,0038 & $-0,0003$ & 0,0038 \\
\hline$b_{M}$ & --- & --- & 0,0054 & 0,0035 & 0,0054 & 0,0035 \\
\hline$\Delta x_{D}$ & 25,8714 & 0,0207 & 25,8506 & 0,0279 & 25,8508 & 0,0280 \\
\hline$\Delta y_{D}$ & 43,4734 & 0,0175 & 43,4143 & 0,0232 & 43,2825 & 0,1190 \\
\hline$\theta_{D}$ & 0,0034 & 0,0007 & 0,0039 & 0,0008 & 0,0039 & 0,0008 \\
\hline$S D$ & --- & ---- & 0,0019 & 0,0007 & $-0,0046$ & 0,0059 \\
\hline$b_{D}$ & --- & ---- & 0,0010 & 0,0052 & 0,0011 & 0,0052 \\
\hline$k_{l}$ & ---- & ---- & --- & --- & $2,60 \times 10^{-6}$ & $2,20 \times 10^{-6}$ \\
\hline$k_{2}$ & --- & --- & --- & --- & $4,19 \times 10^{-10}$ & $3,41 \times 10^{-10}$ \\
\hline
\end{tabular}

A partir da análise da Tabela 4 observa-se que no experimento 3 os POIs $b_{E}$ e $b_{D}$ apresentaram desvios-padrão maior que os seus valores estimados. O mesmo ocorreu com os POIs $s_{E}, b_{E}, s_{D} \mathrm{e}$ $b_{D}$ no experimento 4. Deste modo, as calibrações nos experimentos 3 e 4 foram realizadas novamente com aplicações de injunções absolutas nos POIs citados, atribuindo valores iguais à zero. 
Posteriormente à nova estimativa dos POEs e dos POIs e aplicação dos mesmos nas Equações de Colinearidade inversas, a avaliação da exatidão das orientações foi realizada. Os valores de $Z_{i}$ nas equações foram obtidos dos próprios pontos de verificação. Com a finalidade de se obter uma representação mais fiel da situação dos deslocamentos na superfície terrestre, as coordenadas dos pontos de verificação foram transformadas de coordenadas plano-retangulares do sistema UTM (E, N) para coordenadas do Sistema Geodésico Local (SGL) $\left(X_{L}, Y_{L}\right)$. A Tabela 5 apresenta a Raiz do Erro Quadrático Médio (REQM) nas componentes $X_{L}$ e $Y_{L}$ em todos os experimentos realizados.

Tabela 5: REQM das componentes planimétricas dos pontos de verificação nos experimentos.

\begin{tabular}{c|c|c}
\hline Experimentos & REQM X $_{\mathbf{L}}(\mathbf{m})$ & REQM Y $_{\mathbf{L}}(\mathbf{m})$ \\
\hline 1 & 5,89 & 10,82 \\
\hline 2 & 4,44 & 3,76 \\
\hline 3 & 4,41 & 3,64 \\
\hline 4 & 4,34 & 3,62 \\
\hline
\end{tabular}

De uma forma geral, ao observar-se os dados da Tabela 5, nota-se que há uma diminuição nos valores de REQM em ambas as componente $Y_{L}$ e $X_{L}$ quando se realiza a calibração geométrica em órbita (comparação do experimento 1 com os demais). Analisando o experimento 2, com a consideração apenas das translações e rotações das matrizes de $\mathrm{CCD}$ em relação às posições nominais no plano focal, as melhorias em acurácia chegam a $1,45 \mathrm{~m}$ na componente $X_{L}$ e 7,06 m na componente $Y_{L}$. Considerando a adição da modelagem do fator de escala na direção do eixo $y_{c}$ juntamente com a modelagem da flexão das matrizes de CCD (experimento 3 ) no grupo de POIs do experimento anterior, as melhorias foram praticamente as mesmas do experimento 2 , em ambas as componentes comparando-se aos resultados do experimento 1. Por fim, o experimento 4 que considera além dos POIs contidos no grupo do experimento 3 a modelagem da distorção radial simétrica do sistema de lentes do sensor, apresentou melhorias de exatidão planimétrica em mais de 1,5 m em $X_{L}$ e de 7,2 $\mathrm{m}$ em $Y_{L}$ comparando-se com os valores do experimento 1 . Percebe-se que as melhorias foram praticamente iguais às dos experimentos 2 e 3. Também notase que em todos os experimentos de calibração a melhora da exatidão na componente $Y_{L}$ em relação ao experimento 1 é maior do que o da componente $X_{L}$. A Figura 9 apresenta os resultados de todos os experimentos em termos de exatidão planimétrica resultante.

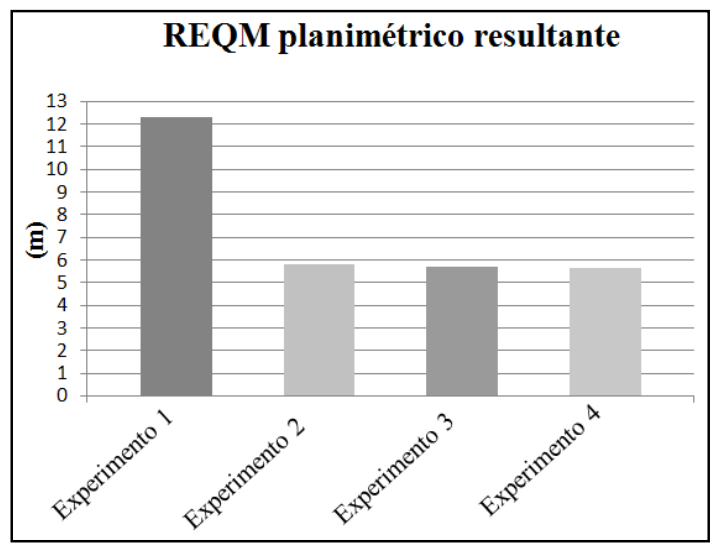

Figura 9: REQM planimétricos resultantes dos pontos de verificação nos experimentos.

Com o objetivo de analisar a qualidade da determinação física dos POIs em todos os experimentos de calibração, foram realizadas verificações de correlações entre os POIs 
estimados nos experimentos 2, 3 e 4, a partir da MVC dos parâmetros ajustados. Neste estudo foram considerados como altamente correlacionados o par de parâmetros cujo coeficiente de correlação assumiu valores acima de $70 \%(\rho=0,7)$. As Tabela 6,7 e 8 apresentam os coeficientes de correlação entre os POIs nos experimentos 2, 3 e 4 respectivamente.

Tabela 6: Coeficientes de correlação entre os POIs no experimento 2.

\begin{tabular}{c|rrrrrr}
\hline & \multicolumn{1}{|c}{$\Delta x_{E}$} & \multicolumn{1}{c}{$\Delta y_{E}$} & \multicolumn{1}{c}{$\theta_{E}$} & $\Delta x_{D}$ & $\Delta y_{D}$ & $\theta_{D}$ \\
\hline$\Delta x_{E}$ & 1.00 & & & & & \\
$\Delta y_{E}$ & 0.08 & 1.00 & & & & \\
$\theta_{E}$ & -0.60 & -0.74 & 1.00 & & & \\
$\Delta x_{D}$ & 0.90 & -0.25 & -0.21 & 1.00 & & \\
$\Delta y_{D}$ & 0.45 & -0.01 & -0.59 & 0.16 & 1.00 & \\
$\theta_{D}$ & -0.73 & -0.68 & 0.98 & -0.38 & -0.55 & 1.00 \\
\hline
\end{tabular}

Tabela 7: Coeficientes de correlação entre os POIs no experimento 3.

\begin{tabular}{c|ccccccccc}
\hline & \multicolumn{1}{c}{$\Delta x_{E}$} & $\Delta y_{E}$ & $\theta_{E}$ & $s_{E}$ & $b_{M}$ & $\Delta x_{D}$ & $\Delta y_{D}$ & $\theta_{D}$ & $s_{D}$ \\
\hline$\Delta x_{E}$ & 1.00 & & & & & & & & \\
$\Delta y_{E}$ & -0.25 & 1.00 & & & & & & & \\
$\theta_{E}$ & -0.68 & -0.49 & 1.00 & & & & & & \\
$s_{E}$ & -0.40 & 0.72 & 0.00 & 1.00 & & & & & \\
$b_{M}$ & -0.80 & -0.35 & 0.88 & -0.17 & 1.00 & & & & \\
$\Delta x_{D}$ & 0.90 & -0.58 & -0.31 & -0.44 & -0.56 & 1.00 & & & \\
$\Delta y_{D}$ & 0.64 & -0.67 & -0.22 & -0.96 & -0.10 & 0.64 & 1.00 & & \\
$\theta_{D}$ & -0.71 & -0.47 & 1.00 & -0.02 & 0.92 & -0.35 & -0.20 & 1.00 & \\
$s_{D}$ & -0.44 & 0.93 & -0.35 & 0.54 & -0.08 & -0.77 & -0.58 & -0.31 & 1.00 \\
\hline
\end{tabular}

Tabela 8: Coeficientes de correlação entre os POIs no experimento 4.

\begin{tabular}{c|rrrrrrrrr}
\hline & \multicolumn{1}{c}{$\Delta x_{E}$} & $\Delta y_{E}$ & $\theta_{E}$ & $b_{M}$ & $\Delta x_{D}$ & $\Delta y_{D}$ & $\theta_{D}$ & $k_{1}$ & $k_{2}$ \\
\hline$\Delta x_{E}$ & 1.00 & & & & & & & & \\
$\Delta y_{E}$ & -0.05 & 1.00 & & & & & & & \\
$\theta_{E}$ & -0.69 & -0.62 & 1.00 & & & & & & \\
$b_{M}$ & -0.80 & -0.54 & 0.88 & 1.00 & & & & & \\
$\Delta x_{D}$ & 0.90 & -0.39 & -0.30 & -0.56 & 1.00 & & & & \\
$\Delta y_{D}$ & 0.62 & -0.74 & -0.06 & -0.03 & 0.72 & 1.00 & & & \\
$\theta_{D}$ & -0.72 & -0.62 & 1.00 & 0.91 & -0.35 & -0.06 & 1.00 & & \\
$k_{1}$ & 0.41 & -0.81 & 0.38 & 0.07 & 0.77 & 0.68 & 0.33 & 1.00 & \\
$k_{2}$ & -0.31 & 0.66 & -0.43 & -0.01 & -0.70 & -0.43 & -0.36 & -0.95 & 1.00 \\
\hline
\end{tabular}

A partir das Tabelas 6, 7 e 8 observa-se que há ocorrências de altas correlações entre certos POIs e até casos de completa correlação, como por exemplo entre $\theta_{E}$ e $\theta_{D}$. A partir destes resultados verifica-se que o significado físico da estimativa dos POIs altamente correlacionados com outros foi perdido. A fim de investigar se os POIs completamente correlacionados com outros $(\rho=1)$ 
podiam ser desconsiderados, ou seja, uma simplificação do modelo, os experimentos de calibração foram realizados novamente, retirando estes POIs do conjuntos de parâmetros. Como resultado obteve-se uma diminuição da exatidão em ambas as componentes $X_{L}$ e $Y_{L}$, com maiores valores de REQM em $Y_{L}$. Deste modo, considerou-se os experimentos sem retirar os POIs completamente correlacionados com outros.

Com a finalidade de se investigar a usabilidade dos POIs estimados nos experimentos 2,3 e 4 na orientação de outras imagens HRC, os coeficientes de correlação entre os POIs e os POEs, calculados a partir da MVC dos parâmetros ajustados, foram analisados. A Tabela 9 apresenta os resultados.

Tabela 9: Coeficientes de correlação entre POIs e POEs nos experimentos 2, 3 e 4.

\begin{tabular}{|c|c|c|c|c|c|c|c|c|c|c|c|}
\hline \multicolumn{12}{|c|}{ Experimento 2} \\
\hline & $X_{0}$ & $u_{x}$ & $Y_{0}$ & $u_{y}$ & $\bar{Z}_{0}$ & $u_{z}$ & $\omega$ & $\varphi$ & $\kappa_{0}$ & $d_{1}$ & $d_{2}$ \\
\hline$\Delta x_{E}$ & -0.26 & 0.22 & 0.28 & -0.34 & -0.93 & -0.63 & 0.79 & 0.94 & 0.94 & 0.12 & 0.20 \\
\hline$\Delta y_{E}$ & -0.69 & -0.34 & -0.52 & 0.47 & -0.30 & 0.27 & -0.05 & 0.37 & 0.37 & -0.17 & 0.50 \\
\hline$\theta_{E}$ & 0.89 & -0.11 & 0.52 & 0.07 & 0.61 & 0.40 & -0.16 & -0.69 & -0.69 & 0.15 & -0.79 \\
\hline$\Delta x_{D}$ & 0.18 & 0.13 & 0.66 & -0.31 & -0.85 & -0.49 & 0.92 & 0.81 & 0.81 & 0.24 & -0.22 \\
\hline$\Delta y_{D}$ & -0.71 & 0.71 & -0.55 & -0.63 & -0.16 & -0.86 & -0.18 & 0.23 & 0.23 & -0.24 & 0.83 \\
\hline$\theta_{D}$ & 0.80 & -0.11 & 0.36 & 0.09 & 0.74 & 0.44 & -0.34 & -0.81 & -0.81 & 0.10 & -0.69 \\
\hline \multicolumn{12}{|c|}{ Experimento 3} \\
\hline & $X_{0}$ & $u_{x}$ & $Y_{0}$ & $u_{y}$ & $Z_{0}$ & $u_{i}$ & $\omega$ & $\varphi$ & $\mathrm{K}_{0}$ & $d_{l}$ & $d_{2}$ \\
\hline$\Delta x_{E}$ & -0.25 & 0.36 & 0.24 & -0.46 & -0.79 & -0.84 & 0.67 & 0.80 & 0.80 & 0.05 & 0.27 \\
\hline$\Delta y_{E}$ & -0.39 & -0.42 & -0.29 & 0.42 & -0.32 & 0.25 & 0.09 & 0.35 & 0.35 & 0.00 & 0.01 \\
\hline$\theta_{E}$ & 0.71 & -0.11 & 0.24 & 0.16 & 0.83 & 0.63 & -0.46 & -0.89 & -0.89 & 0.04 & -0.49 \\
\hline$S_{E}$ & 0.36 & -0.65 & 0.37 & 0.54 & -0.22 & 0.60 & 0.36 & 0.17 & 0.17 & 0.24 & -0.68 \\
\hline$b_{M}$ & 0.30 & -0.02 & -0.24 & 0.13 & 0.99 & 0.58 & -0.82 & -1.00 & -1.00 & -0.11 & -0.07 \\
\hline$\Delta x_{D}$ & 0.14 & 0.35 & 0.52 & -0.47 & -0.59 & -0.69 & 0.67 & 0.56 & 0.56 & 0.13 & -0.01 \\
\hline$\Delta y_{D}$ & -0.39 & 0.65 & -0.25 & -0.59 & -0.06 & -0.76 & -0.10 & 0.11 & 0.11 & -0.19 & 0.66 \\
\hline$\theta_{D}$ & 0.65 & -0.10 & 0.17 & 0.17 & 0.87 & 0.64 & -0.52 & -0.92 & -0.92 & 0.01 & -0.43 \\
\hline$S D$ & -0.52 & -0.32 & -0.57 & 0.41 & -0.02 & 0.30 & -0.26 & 0.07 & 0.08 & -0.13 & 0.22 \\
\hline \multicolumn{12}{|c|}{ Experimento 4} \\
\hline & $X_{0}$ & $u$ & $Y_{0}$ & & $Z_{0}$ & $\bar{u}$ & $\omega$ & $\varphi$ & $\kappa$ & $d_{l}$ & $d_{2}$ \\
\hline$\Delta x_{E}$ & -0.28 & 0.40 & 0.22 & -0.47 & -0.79 & -0.88 & 0.66 & 0.80 & 0.80 & 0.04 & 0.16 \\
\hline$\Delta y_{E}$ & -0.39 & -0.46 & -0.17 & 0.40 & -0.52 & 0.18 & 0.29 & 0.54 & 0.54 & 0.04 & 0.14 \\
\hline$\theta_{E}$ & 0.73 & -0.02 & 0.26 & 0.09 & 0.82 & 0.54 & -0.44 & -0.87 & -0.87 & 0.03 & -0.52 \\
\hline$b_{M}$ & 0.32 & -0.04 & -0.23 & 0.15 & 0.99 & 0.61 & -0.82 & -1.00 & -1.00 & -0.10 & -0.07 \\
\hline$\Delta x_{D}$ & 0.13 & 0.47 & 0.51 & -0.55 & -0.59 & -0.81 & 0.67 & 0.57 & 0.56 & 0.11 & -0.17 \\
\hline$\Delta y_{D}$ & -0.17 & 0.62 & -0.06 & -0.57 & -0.01 & -0.69 & -0.04 & 0.04 & 0.04 & -0.12 & 0.31 \\
\hline$\theta_{D}$ & 0.67 & -0.03 & 0.19 & 0.10 & 0.86 & 0.57 & -0.51 & -0.91 & -0.91 & 0.01 & -0.45 \\
\hline$k_{1}$ & 0.59 & 0.45 & 0.65 & -0.50 & 0.00 & -0.43 & 0.32 & -0.06 & -0.06 & 0.13 & -0.49 \\
\hline$k_{2}$ & -0.78 & -0.31 & -0.84 & 0.40 & 0.08 & 0.31 & -0.48 & 0.00 & 0.00 & -0.22 & 0.72 \\
\hline
\end{tabular}

Ao observar-se a Tabela 9 nota-se que quase todos os POIs nos diferentes experimentos apresentam correlação alta ou completa com um grupo de POEs. Desta forma, o uso dos POIs estimados nos experimentos 2, 3 e 4 fica restrito a imagem utilizada no processo. As ocorrências de altas correlações estão relacionadas com as fracas injunções aplicadas nos POEs. Caso as efemérides GPS estivessem disponíveis, os desvios-padrão para as ponderações nas injunções seriam menores, o que possivelmente ocasionaria a redução das correlações. Outro ponto a ser considerado é que a calibração foi feita inevitavelmente com apenas uma imagem, ao passo que se houvessem duas ou mais imagens convergentes, as correlações seriam também reduzidas.

\section{Conclusões e recomendações}

Bol. Ciênc. Geod., sec. Artigos, Curitiba, v. 22, no1, p.108 -131, jan-mar, 2016. 
Os experimentos realizados mostram que a estimativa dos POIs nas orientações (calibração geométrica em órbita) acarreta em uma diminuição nos REQM planimétricos, principalmente na componente $Y_{L}$ do SGL. Este último fato ocorreu por considerar o desalinhamento da matriz de CCD central em relação às demais matrizes de CCD no plano focal do sensor.

Particularmente em relação à aplicação dos diferentes grupos de POIs nas calibrações, os resultados de acurácia planimétrica resultantes apresentaram-se sem variações significativas. $\mathrm{O}$ grupo de POIs considerando as translações, rotações, fatores de escala na direção $y_{c}$ e as flexões das matrizes de CCD juntamente com a modelagem da distorção radial simétrica das lentes, apresentou melhores resultados de exatidão planimétrica do que os demais experimentos de calibração, em comparação com o experimento sem calibração. Com relação ao resultado da exatidão planimétrica do experimento considerando somente os POIs utilizados no experimento 4 de Zhang e Zheng (2012), os valores mostraram-se concordantes em ordem de grandeza, com valores ligeiramente melhores nas componentes $X_{L}$ e $Y_{L}$.

Mesmo com a melhoria em exatidão planimétrica decorrente dos processos de calibração, os resultados em geral não chegaram a atingir o nível sub-pixel. Pode-se relacionar este fato com imprecisões nas medidas dos pontos de controle na imagem, possivelmente devido à baixa qualidade radiométrica da mesma. Esta questão ocorre tanto por conta do pequeno intervalo dinâmico dos níveis de cinza, quanto pela questão do valor do EIFOV (Effective Instantaneous Field of View) do sensor se apresentar mais alto do que o teórico (Carvalho et al., 2009). A influência deste problema em medições de pontos para orientações de imagens HRC pode ser vista, por exemplo, em Marcato Júnior (2011) e Rodrigues e Ferreira (2013). Esta questão também explica o fato de não ter havido resultados sub-pixel em nenhuma das calibrações geométricas em órbita realizadas em Yue et al. (2009) e Zhang e Zheng (2012).

Com relação à análise da qualidade da estimativa física dos POIs, em todos os experimentos de calibração houveram ocorrências de altas correlações entre pares de POIs e de até completas correlações. Conclui-se, neste caso, que o significado físico dos POIs que apresentaram altas correlações com outros POIs foi perdido. Com o objetivo de investigar a necessidade de manter nos experimentos o grupo de POIs que apresentaram correlação completa $(\rho=1$ ou $\rho=-1)$ com outros POIs, analisou-se a eliminação dos mesmos nos experimentos de 2 a 4 . Em outras palavras, testou-se a simplificação dos modelos de calibração sem o POIs completamente correlacionados com outros POIs. Como resultado observou-se em todos os experimentos um aumento nos valores das componentes $X_{L}$ e $Y_{L}$ do REQM, e consequentemente uma diminuição da exatidão planimétrica. Desta forma, decidiu-se manter todos os POIs considerados nos experimentos, uma vez que os mesmos mostraram-se ser realmente importantes em termos de exatidão planimétrica, mesmo tendo completas correlações com outros POIs.

Analisou-se ainda que houveram ocorrências de altas correlações também entre os POIs e os POEs em todos os experimentos de calibração. Deste modo, tem-se que o uso dos POIs estimados neste estudo, não é recomendado em orientações de outras imagens HRC, ou seja, fica restrito à orientação da imagem utilizada neste estudo. As razões possíveis para as altas correlações são a fraca injunção aplicada nos componentes do vetor de estado inicial e a impossibilidade do uso de estéreo imagens convergentes.

Recomenda-se para trabalhos futuros realizar calibrações geométricas em órbita utilizando modelos de plataforma polinomiais, com as estruturas das classes de modelos Posição-Rotação e Órbita-Atitude. 


\section{AGRADECIMENTOS}

Os autores agradecem ao IBGE pela disponibilização das ortofotos utilizadas para a coleta dos pontos e ao Setor de Atendimento ao Usuário do INPE (ATUS - INPE) por ceder as imagens HRC-CBERS 2B em nível de processamento 1.

\section{REFERÊNCIAS BIBLIOGRÁFICAS}

Baltsavias, E., Zhang L., and Eisenbeiss, H. "DSM generation and interior orientation determination of IKONOS images using a Testfield in Switzerland." Photogramm. Fernerkund Geoinf 1 (2006): 41-54.

Brown, D. C. "Close-range camera calibration" Photogrammetric Engineering 37 (1971): 855866.

Carvalho, L. A. S., Strauss C. and Fonseca, L. M. G. "Determinação da resolução efetiva da câmera HRC-CBERS-2B pelo método de espalhamento de borda". Paper presented at the XIV Simpósio Brasileiro de Sensoriamento Remoto, Natal, Rio Grande do Norte, April 25-30, 2009.

Dowman, I., and Michalis, P. "Generic rigorous model for along track stereo satellite sensors". Paper presented at the ISPRS workshop on High resolution mapping from Space, Hanover, Niedersachsen, October 4-6, 2003.

Dowman, I., Michalis, P., and Li, L. "Analysis of Urban Landscape Using Multi Sensor Data”. Paper presented at the $4^{\text {th }}$ ALOS PI Symposium, Tokyo, Japan, November 15-17, 2010.

Epiphanio, J. C. N. "CBERS: estado atual e futuro". Paper presented at the XIV Simpósio Brasileiro de Sensoriamento Remoto, Natal, Rio Grande do Norte, April 25-30, 2009.

Gachet, R. "Spot5 In-Flight Commissioning: Inner orientation of HRG and HRS instruments". Paper presented at the ISPRS Congress, Istanbul, Turkey, July 12-23, 2004.

Gruen A., Kocaman, S., and Wolf, K.. "Calibration and Validation of Early ALOS/PRISM Images". The Journal of the Japan Society of Photogrammetry and Remote Sensing 46 (2007): $24-38$ Accessed October 01, 2010.

Gugan, D. and Dowman, I. "Topographic mapping from SPOT imagery" Photogrammetric Engineering \& Remote Sensing 54 (1988): 1409-14. Accessed September 21, 2014. doi: 10.1111/j.1477-9730.1987.tb00581.x.

INPE. "Satélite Sino-Brasileiro de Recursos Terrestres". Accessed October, 10, 2014. http://www.cbers.inpe.br.

Jacobsen, K. "Calibration of IRS-1C PAN-camera". Paper presented at the Sensors and Mapping from Space, Hannover, Niedersachsen, 1997. Accessed September 14, 2014.

Jacobsen, K."Geometry of satellite images - calibration and mathematical models". Paper presented at the ISPRS international conference on Korean Society of Remote Sensing, Jeju, 2005.

Kim, T., and Dowman, I. "Comparison of two physical sensor models for satellite images: Position-Rotation model and Orbit-Attitude model". The Photogrammetric Record 21 (2006): 110-23. Acessed May 23, 2009. doi: 10.1111/j.1477-9730.2006.00363.x.

Kocaman, S. "Sensor modeling and validation for linear array aerial and satellite imagery" $\mathrm{PhD}$ diss., Institut f. Geodäsie u. Photogrammetrie, 2007. 
Kornus, W., Lehner, M., and Schroeder, M. "Geometric Inflight Calibration of the Stereoscopic CCD-Linescanner MOMS-2P". Paper presented at the ISPRS Symposium on Earth Observation System for Susatinable Development Technical Commission I: Sensors, Platforms and Imagery, Bangalore, India, February, 2-27, 1998.

Machado, M. V.; Rodrigues T. L., and Debiasi, P. "Orientação de imagens HRC pelo Modelo de Kepler com parâmetros adicionais da geometria interna do sensor". Paper present at the VIII Colóquio Brasileiro de Ciências Geodésicas, Curitiba, Paraná, December, 2-5, 2013.

Marcato Junior, J. "Fototriangulação em bloco de imagens orbitais com modelos rigorosos baseados em pontos e retas." PhD diss., Universidade Estadual Paulista, 2011.

Marcato Junior, J., and Tommaselli, A. M. G. "Exterior orientation of CBERS-2B imagery using multi-feature control and orbital data". ISPRS Journal of Photogrammetry and Remote Sensing 79 (2013): 219-25. Acessed January 27, 2015. doi: 10.1016/j.isprsjprs.2013.02018.

Marcato Junior, J. et al., "Orientação de imagens CBERS-2B usando o modelo rigoroso de colinearidade com dados orbitais". Boletim de Ciências Geodésicas 17 (2011): 401- 16. Acessed November 11, 2012. doi: 10.1590/S1982-21702011000300005.

Michalis, P. "Generic rigorous model for along track stereo satellite sensors." PhD diss., University College London, 2005.

Michalis, P., and Dowman, I. "A rigorous model and DEM generation for SPOT5-HRS". Paper presented at the XX ISPRS Congress, Comission I, Istanbul, Turkey, July 12-23, 2004.

Michalis, P., and Dowman, I. “A Generic Model for Along Track Stereo Sensors Using Rigorous Orbit Mechanics" Photogrammetric Engineering and Remote Sensing 74 (2008): 303-09. Accessed July 13, 2011. doi: 10.14358/PERS.74.3.303.

Michalis, P., and Dowman, I. "Noise-Reduction and exterior orientation improvement by the coplanarity equation for ALOS-PRISM". Paper presented at the $4^{\text {th }}$ ALOS PI Symposium, Tokyo, Japan, November 15-17, 2010.

Michalis, P.; and Dowman, I. "A model for along track stereo sensors using rigorous orbit mechanics". International Archives of the Photogrammetry, Remote Sensing and Spatial Information Sciences 36 (2005).

Michalis, P., and Dowman, I. "An improved model for along-track stereo sensors using rigorous orbit mechanics and navigation data". Paper presented at the ISPRS Commission I Symposium on From sensors to imagery, Paris, France, May 04-06, 2006.

Mulawa, D. "On-Orbit geometric calibration of the Orbview-3 high resolution imaging satellite". Paper present at the XX ISPRS Congress on Geo-Imagery Bridging Continents, Istanbul, Turkey, July 12-23, 2004.

Orun, A. B, and Natarajan, K. "A Modified Bundle Adjustment Software for SPOT Imagery and Photography: Tradeoff”. Photogrammetric Engineering and Remote Sensing 60 (1994): 1431-37.

Poli, D. "Modelling of Spaceborne Linear Array Sensors." PhD diss., Institute of Geodesy and Photogrammetry, 2005.

Radhadevi, P. V., and Solanki, S. S. "In-Flight geometric calibration of different cameras of IRS P6 using a physical sensor model". The Photogrammetric Record 23 (2008): 69 - 89. Accessed January 13, 2015. doi: 10.1111/j.1477-9730.2007.00453.x

Radhadevi, P. V, Sasikumar, T.P., and Ramachandran, R. "Orbit attitude modelling and derivation of ground co-ordinates from spot stereopairs". Journal of Photogrammetry and 
Remote Sensing 49 (1994): 22-28. Accessed April 17, 2015. doi: 10.1016/0924-2716(94)900434.

Rodrigues, T. L., and Ferreira, L. D. D. "Aplicação do movimento kepleriano na orientação de imagens HRC - CBERS 2B". Boletim de Ciências Geodésicas 19 (2013): 114-34. Accessed May 1, 2013. doi: 10.1590/S1982-21702013000100008.

Rodrigues, T. L., Mitishita E.A., and Damasceno F. D. "Adaptação do Modelo de Kepler e inserção do harmônico zonal J2 do geopotencial na orientação de imagens orbitais". Paper present at the VIII Colóquio Brasileiro de Ciências Geodésicas, Curitiba, Paraná, December, 2-5, 2013.

Seeber, G. Satellite Geodesy: foundations, methods, and applications. Berlim: Walter de Gruyter, 2003.

Tadono T., et al. "Calibration and Validation of PRISM Onboard ALOS". Paper present at the XX ISPRS Congress on Geo-Imagery Bridging Continents, Istanbul, Turkey, July 12-23, 2004.

Toutin, T. "Geometric processing of Remote Sensing images: models, algorithms and methods" International Journal of Remote Sensing 25 (2004): 1893-924. Accessed April 13, 2014. doi: 10.1080/0143116031000101611.

Toutin, T., et al. "In-flight calibration of SPOT-5 and Formosat-2". Paper presented at the ISPRS Commission I Symposium on From sensors to imagery, Paris, France, May 04-06, 2006.

Wang, M. et al., "On-Orbit geometric calibration model and its applications for high-resolution optical satellite imagery". Remote Sensing 6 (2014): 4391-4408. Accessed January 10, 2015. doi: $10.3390 /$ rs6054391.

Weser, T. et al., "Development and testing of a generic sensor model for pushbroom satellite imagery". The Photogrammetric Record 23 (2008): 255-274. Accessed December 15, 2014. doi: 10.1111/j.1477-9730.2008.00489.x.

Yue, Q. et al. "The adjustment of CBERS-02B pan image". Remote Sensing for Land \& Resources 3 (2009): 61-63. Accessed December 16, 2014.

Zhang, Y., and Zheng, M. "Bundle Block Adjustment with self-calibration of long orbit CBERS02B imagery". Paper presented at the ISPRS Congress, Melbourne, Australia, 25 August-01 September, 2012. 